\title{
Initial report of decreased SARS-CoV-2 viral load after inoculation with the BNT162b2 vaccine
}

\author{
Matan Levine-Tiefenbrun ${ }^{1,6}$, Idan Yelin ${ }^{1,6 \bowtie}$, Rachel Katz ${ }^{2}$, Esma Herzel ${ }^{2}$, Ziv Golan ${ }^{3}$, \\ Licita Schreiber ${ }^{3}$, Tamar Wolf ${ }^{3}$, Varda Nadler ${ }^{3}$, Amir Ben-Tov ${ }^{2,4}$, Jacob Kuint ${ }^{2,4}$, Sivan Gazit ${ }^{2}$, \\ Tal Patalon², Gabriel Chodick $\mathbb{D}^{2,4}$ and Roy Kishony ${ }^{1,5} \bowtie$
}

\begin{abstract}
Beyond their substantial protection of individual vaccinees, coronavirus disease 2019 (COVID-19) vaccines might reduce viral load in breakthrough infection and thereby further suppress onward transmission. In this analysis of a real-world dataset of positive severe acute respiratory syndrome coronavirus 2 (SARS-CoV-2) test results after inoculation with the BNT162b2 messenger RNA vaccine, we found that the viral load was substantially reduced for infections occurring 12-37 d after the first dose of vaccine. These reduced viral loads hint at a potentially lower infectiousness, further contributing to vaccine effect on virus spread.
\end{abstract}

The recently authorized BNT162b2 Coronavirus Disease 2019 (COVID-19) messenger RNA (mRNA) vaccine is approximately $95 \%$ efficient in preventing polymerase chain reaction (PCR)-confirmed symptomatic disease from $7 \mathrm{~d}$ after the second dose and also provides some early protection starting $12 \mathrm{~d}$ after the first dose $\mathrm{e}^{1,2}$. As countries race to vaccinate a substantial portion of their populations in the coming months, it is hoped that the basic reproduction number of the virus will decrease. This effect can be achieved by reducing the number of susceptible people, as well as by reducing viral load and, thereby, viral shedding of post-vaccination infections, which might render them less infectious ${ }^{3-7}$. However, the effect of vaccination on viral load in COVID-19 post-vaccination infections is currently unknown ${ }^{8}$.

As of February 11, 2021, Maccabi Healthcare Services (MHS) in Israel has vaccinated more than 1 million of its members as part of a national rapid rollout of the vaccine. MHS member SARS-CoV-2 tests are often carried out in the MHS central laboratory, which offers the opportunity to track post-vaccination infections. In this study, we retrospectively collected and analyzed the quantitative reverse transcription PCR (RT-qPCR) test measurements of three SARS-CoV-2 genes-E, $N$ and RdRp (Allplex 2019-nCoV assay, Seegene)-from positive post-vaccination tests performed at the MHS central laboratory between December 21, 2020, and February 11, 2021 ( $n=4,938$ patients, study population; Table 1$)$. The study period was characterized by high and steady rates of positive COVID-19 tests (Extended Data Fig. 1), indicating an ongoing epidemic wave.

In an analysis of the infection cycle threshold (Ct) over time, we found that the mean viral load substantially decreased $12 \mathrm{~d}$ after vaccination with the first vaccine dose, coinciding with the known early onset of vaccine-mediated protection ${ }^{1}$. When we calculated the mean $\mathrm{Ct}$ for post-vaccination infections identified on each day
Table 1 | Study population

\begin{tabular}{|llll} 
Age group (years) & Total no. of patients & Male & Female \\
\hline $16-19$ & 241 & 143 & 98 \\
\hline $20-29$ & 425 & 216 & 209 \\
\hline $30-39$ & 485 & 277 & 208 \\
\hline $40-49$ & 1,077 & 513 & 564 \\
\hline $50-59$ & 1,344 & 708 & 636 \\
\hline $60-69$ & 821 & 445 & 376 \\
\hline $70-79$ & 422 & 216 & 206 \\
\hline $80-89$ & 123 & 53 & 70 \\
\hline
\end{tabular}

after vaccination, we found that the $\mathrm{Ct}$ values of positive samples collected $12-37 \mathrm{~d}$ after vaccination with the first dose (a second dose having been given on day 21 for all samples taken after day 21) were higher than the $\mathrm{Ct}$ values of positive samples taken during the first $11 \mathrm{~d}$ after vaccination for the $R d R p$ gene (Fig. 1) and for the genes $N$ and $E$ (Extended Data Fig. 2) $\left(P<10^{-19}\right.$ for the three genes, Mann-Whitney $U$-test). Differences in mean Ct calculated for these two time periods ranged from $1.7 \pm 0.2$ for $R d R p$ to $1.6 \pm 0.2$ for gene $E$ and $1.4 \pm 0.2$ for gene $N$.

We next compared the Ct values of these post-vaccination infections with $\mathrm{Ct}$ values of positive tests of unvaccinated patients. All of the tests were of MHS members and were carried out at the central laboratory. Given that viral load could be associated with age and sex $^{9}$, we assembled control groups of positive tests in unvaccinated patients with matching age group, sex and sampling date range (Methods). Comparing the post-vaccination positive tests from days 1-11 $(n=3,050)$ with their corresponding demographically and calendrically matched control group of the same size, we found no significant difference in the distribution of $\mathrm{Ct}$ values for $R d R p$ (Fig. 2a and Extended Data Fig. 4a) or for genes $N$ and $E$ (Extended Data Figs. 3a and 4a). However, in a comparison of the Ct distribution for infections from days $12-37$ after vaccination $(n=1,888)$ with that of demographically matched unvaccinated control group infections $(n=1,888)$, we identified a significant increase in $\mathrm{Ct}$ in vaccinated individuals (Fig. $2 \mathrm{~b}$ for $R d R p$ and Extended Data Fig. $3 \mathrm{~b}$ for genes $N$ and $E$; Mann-Whitney $U$-test, $\mathrm{P}<10^{-10}$ for all three genes; Extended Data Fig. 4b,c). Finally, applied on all of the

${ }^{1}$ Faculty of Biology, Technion - Israel Institute of Technology, Haifa, Israel. ${ }^{2}$ Maccabitech, Maccabi Health Services, Tel Aviv, Israel. ${ }^{3}$ Maccabi Mega-Lab, Maccabi Healthcare Services, Rehovot, Israel. ${ }^{4}$ Sackler Faculty of Medicine, Tel-Aviv University, Tel Aviv, Israel. ${ }^{5}$ Faculty of Computer Science, Technion - Israel Institute of Technology, Haifa, Israel. ${ }^{6}$ These authors contributed equally: Matan Levine-Tiefenbrun, Idan Yelin. ${ }^{\bowtie e-m a i l: ~ i d a n y @ t e c h n i o n . a c . i l ; ~}$ rkishony@technion.ac.il 


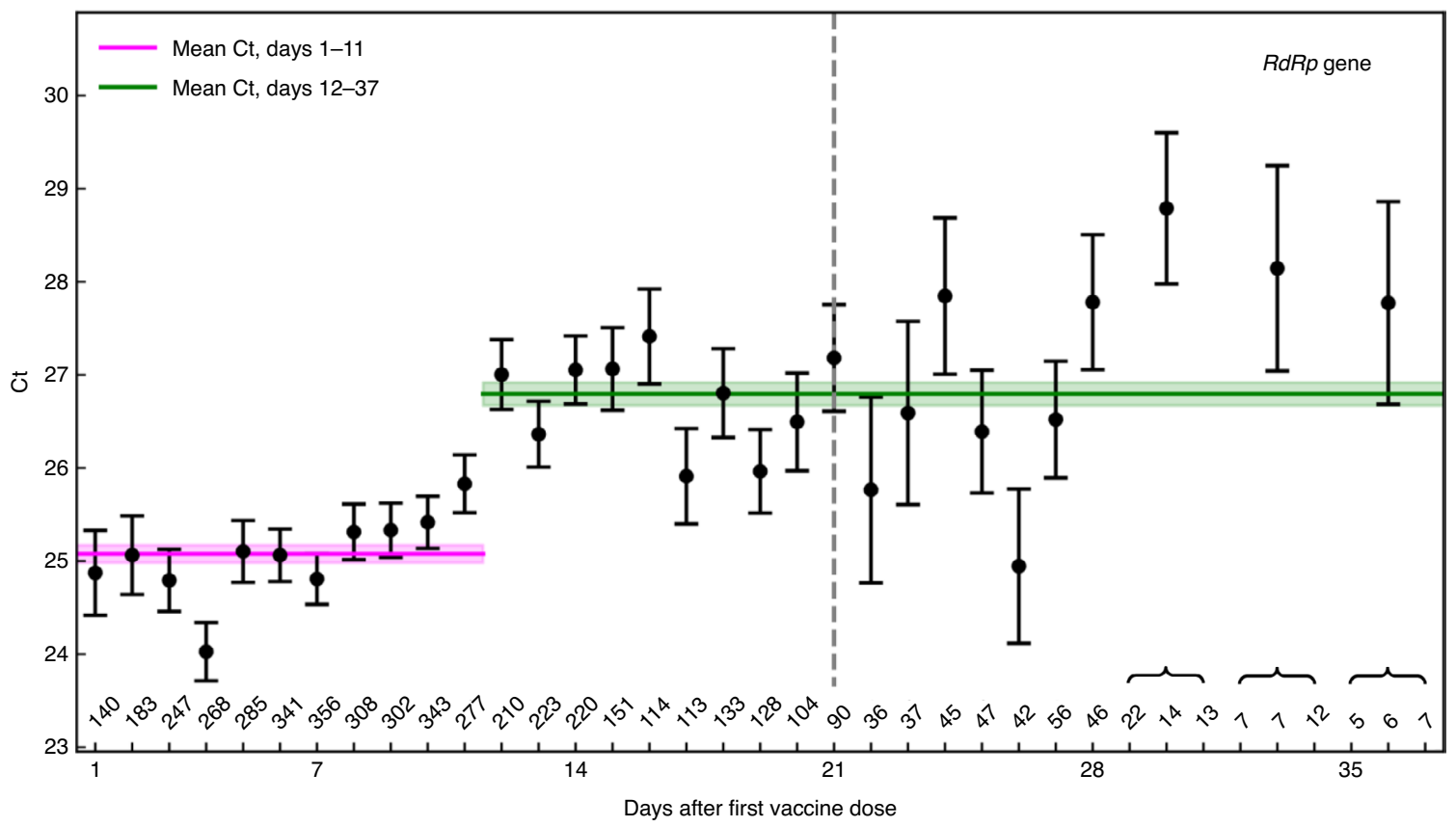

Fig. 1 | Decreased SARS-CoV-2 viral load after $12 \mathrm{~d}$ post-vaccination. Mean Ct values of the RdRp gene for positive tests after vaccination are plotted by the post-vaccination day in which the sample was taken. The dashed line on day 21 indicates inoculation with the second dose. The number of positive test results for each day is indicated below (in total, $n=4,938$ ). Black error bars and green or magenta shading indicate the standard error of the mean. For days 29-37, each dot represents the mean of three consecutive days. For genes $E$ and $N$, see Extended Data Fig. 2.

infections (post-vaccination and matched unvaccinated, $n=9,876$ ), a multivariable linear regression model accounting for age, sex and vaccination found $\mathrm{Ct}$ regression coefficients ranging from 1.51 ( $N$ gene) to $1.76(R d R p)$ for vaccination $12-21 \mathrm{~d}$ before infection sampling and even higher coefficients ranging from 1.90 ( $N$ gene) to $2.16(R d R p)$ for vaccination $22-37 \mathrm{~d}$ before infection sampling (Fig. 2c for RdRp and Extended Data Fig. 5 for $N$ and $E$ genes; similar coefficients for independent models for each time bin are shown in Extended Data Fig. 6). Given that a difference of $1 \mathrm{Ct}$ unit is approximately equivalent to a factor of 2 in the number of viral particles per sample, these $\mathrm{Ct}$ differences represent a decrease of 2.8-4.5-fold in viral load in vaccinated individuals.

Viral load has been shown to be associated with COVID-19 symptomatic disease as well as with time since infection ${ }^{9-12}$. To alleviate potential biases toward asymptomatic and/or long-term infections or persistent shedding in vaccinated participants, we repeated the multivariable linear regression analysis and restricted it to patients for whom a referral for COVID-19 testing was recorded ( $n=783$ vaccinees and the same number of demographically matched unvaccinated controls). For the linear regression model, these referrals were categorized into those indicating COVID-19 symptoms versus other causes for the test, mostly epidemiological contact tracing ( 0 and 1 , asymptomatic and symptomatic). We found that symptomatic disease was indeed correlated with lower Ct values and that, when we adjusted for symptomatic infections, the association of vaccination with $\mathrm{Ct}$ remained (albeit with lower significance due to the reduced sample size of referral-based tests; Extended Data Fig. 7).

The results show that infections occurring $12 \mathrm{~d}$ or longer after vaccination have significantly reduced viral loads at the time of testing, potentially affecting viral shedding and contagiousness as well as the severity of the disease ${ }^{13}$. This report is based on an observational study, not a randomized controlled trial, and has several associated limitations. First, the group of vaccinees might differ from the demographically matched control group in ways that could affect the observed viral load, such as behavior, tendency to get tested and general health status. Second, the different viral variants, which could be associated with different viral loads, might affect different parts of the population. Third, by including only the first positive test for each patient, we attempted to minimize the effect of long-term, low-viral-load infections, but it is still possible that the association we observed, especially in the early post-vaccination days, reflects infections lasting from pre-immunization transmission events ${ }^{9-12}$. The average viral load might, therefore, continue to change in longer post-vaccination times, when infections are more strongly enriched for post-immunization transmissions, or due to change in vaccinee behavior, especially upon obtaining a vaccination certificate. Fourth, given that vaccines prevent symptomatic disease, post-vaccination tests might be enriched for cases of asymptomatic carriage characterized by lower viral load, although we note that the association of vaccination with low viral load remains even when adjusting for symptomatic disease (Extended Data Fig. 7). Finally, the oro-nasopharyngeal test does not distinguish the viral load in the nose from the one in the oral cavity and does not account for virus viability, which would be a better measure of potential infectiousness. Moreover, the infectious dose of SARS-CoV-2 in humans is presently unknown. The accumulation of wider and longer-term datasets, including contact tracing data as well as virus viability and genomics, will allow better quantification of the vaccine effect on infectiousness and its dependence on viral variants and vaccinee behavior. Nevertheless, at least for the conditions tested here, the lower viral loads that we observed could help fine-tune epidemiological models of vaccine effect on the spread of the virus.

\section{Online content}

Any methods, additional references, Nature Research reporting summaries, source data, extended data, supplementary information, acknowledgements, peer review information; details of author contributions and competing interests; and statements of data and code availability are available at https://doi.org/10.1038/ s41591-021-01316-7. 
a

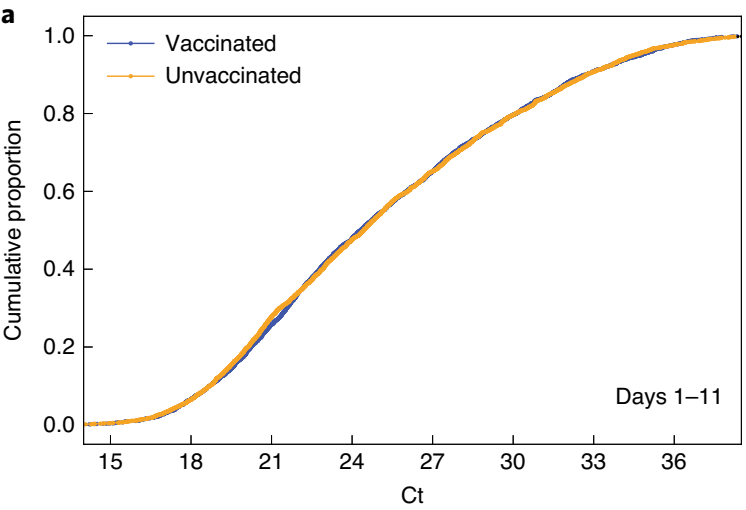

b

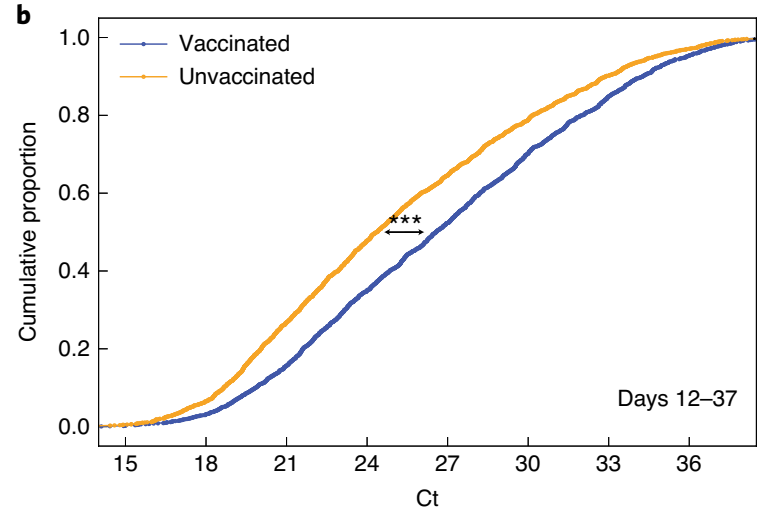

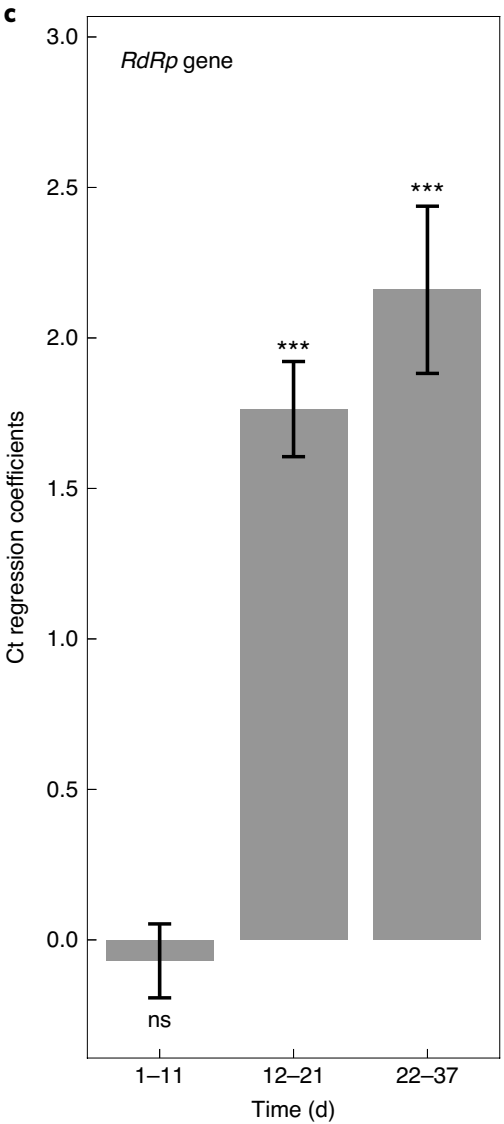

Fig. 2 | Comparison of SARS-CoV-2 viral loads among vaccinated and unvaccinated patients. $\mathbf{a}, \mathbf{b}$, The distribution of $C t$ values of the $R d R p$ gene as determined for positive samples taken 1-11 d after vaccination ( $\mathbf{a}, n=3,050$, blue) and 12-37 d after vaccination $(\mathbf{b}, n=1,888$, blue) with their respective demographically matched control groups (orange, ${ }^{\star \star \star} P<10^{-17}$, two-sided Mann-Whitney U-test). For genes $E$ and $N$, see Extended Data Fig. 3.

c, Coefficient for the association of $\mathrm{Ct}$ of the RdRp gene with vaccination at different vaccination-to-sample time bins in comparison to unvaccinated patients as identified in a single multivariable linear regression analysis accounting for age and sex (Methods; $n=9,876$ ). Error bars represent $1 \mathrm{~s}$.d. ${ }^{\star} P<0.05,{ }^{\star \star} P<0.01$ and ${ }^{\star \star \star} P<0.001$. For genes $E$ and $N$, see Extended Data Fig. 5a,b.

Received: 1 February 2021; Accepted: 9 March 2021;

Published online: 29 March 2021

\section{References}

1. Polack, F. P. et al. Safety and efficacy of the BNT162b2 mRNA Covid-19 vaccine. N. Engl. J. Med. 383, 2603-2615 (2020).

2. Chodcik, G. et al. The effectiveness of the first dose of BNT162b2 vaccine in reducing SARS-CoV-2 infection 13-24 days after immunization: real-world evidence. Preprint at medRxiv https://doi.org/10.1101/2021.01.27.21250612 (2021).

3. Gallagher, M. E. et al. Indirect benefits are a crucial consideration when evaluating SARS-CoV-2 vaccine candidates. Nat. Med. 27, 4-5 (2021).

4. Rubin, E. J. \& Longo, D. L. SARS-CoV-2 vaccination: an ounce (actually, much less) of prevention. N. Engl. J. Med. 383, 2677-2678 (2020).

5. Pollard, A. J. \& Bijker, E. M. A guide to vaccinology: from basic principles to new developments. Nat. Rev. Immunol. 21, 129 (2021).

6. Connors, M., Graham, B. S., Lane, H. C. \& Fauci, A. S. SARS-CoV-2 vaccines: much accomplished, much to learn. Ann. Intern. Med. https://doi. org/10.7326/M21-0111 (2021).

7. Lipsitch, M. \& Dean, N. E. Understanding COVID-19 vaccine efficacy. Science 370, 763-765 (2020).
8. Strategic Advisory Group of Experts on Immunization (SAGE). Interim Recommendations for Use of the Pfizer-BioNTech COVID-19 Vaccine, BNT162b2, Under Emergency Use Listing. https://www.who.int/publications/i/ item/WHO-2019-nCoV-vaccines-SAGE_recommendation-BNT162b2-2021.1 (World Health Organization, 2021).

9. Levine-Tiefenbrun, M. et al. Association of COVID-19 RT-qPCR test false-negative rate with patient age, sex and time since diagnosis. Preprint at medRxiv https://doi.org/10.1101/2020.10.30.20222935 (2020).

10. He, X. et al. Temporal dynamics in viral shedding and transmissibility of COVID-19. Nat. Med. 26, 672-675 (2020).

11. Mina, M. J., Parker, R. \& Larremore, D. B. Rethinking Covid-19 test sensitivity: a strategy for containment. N. Engl. J. Med. 383, e120 (2020).

12. Cevik, M., Marcus, J. L., Buckee, C. \& Smith, T. C. SARS-CoV-2 transmission dynamics should inform policy. Clin. Infect. Dis. https://doi.org/10.1093/cid/ ciaa1442 (2020).

13. Pujadas, E. et al. SARS-CoV-2 viral load predicts COVID-19 mortality. Lancet Respir. Med. 8, e70 (2020).

Publisher's note Springer Nature remains neutral with regard to jurisdictional claims in published maps and institutional affiliations.

(c) The Author(s), under exclusive licence to Springer Nature America, Inc. 2021 


\section{Methods}

Data collection. Anonymized SARS-CoV-2 RT-qPCR Ct values were retrieved for all of the positive post-vaccination samples taken between December 21, 2020 (1 d after the first vaccine was administered) and February 11, 2021, and tested at the MHS central laboratory. Vaccination dates were retrieved from the centralized database of MHS. Patients were excluded if they had a positive sample before vaccination; if they had a positive sample more than $21 \mathrm{~d}$ after the first dose of the vaccine but did not receive the second dose on day 21 ; or if they were over the age of 90 years ( 28 patients older than 90 were not included because it was not possible to match them with unvaccinated controls). For patients with multiple positive post-vaccination tests, only the first test was included. For each test, Ct values for $E$ gene, RdRp gene, $N$ gene and the internal control were determined using Seegene proprietary software for the Allplex 2019-nCoV assay after the standard oro-nasopharyngeal swab specimen collection procedure. The same machine model, the Bio-Rad CFX96 Real-Time PCR Detection System, was used for all of the tests.

Unvaccinated patient control group. As a control group, for each post-vaccination SARS-CoV-2-positive patient, we used an algorithm to randomly choose an unvaccinated positive patient with similar characteristics (same sex and same age (in bins of 10 years)) and a similar date of first positive sample to account for possible calendric trends associated with the national state of the pandemic (nominally up to a 10-d difference, with this difference extended for 168 patients for whom we could not find matching unvaccinated controls) (Table 1).

Statistics. Linear regression. For each viral gene, we calculated the linear regression of $\mathrm{Ct}$ values as a function of sex (0/1, female/male) and age (linear, in years). Adding a quadratic age term was also tested, giving very similar results. Time after vaccination (one-hot encoded binary vector for time bins 1-11 d, 12-21 d and 22-37d; unvaccinated were encoded as all-zero vectors). In an additional multivariable linear regression model, referral for COVID-19 test indicating symptoms was also included (0/1, asymptomatic/symptomatic). For this latter model, only patients for whom the positive test was by referral issued during the four previous days were included. Models were implemented using Python's statsmodels library, version 0.9.0.

Ethics committee approval. The study protocol was approved by the ethics committee of Maccabi Healthcare Services in Tel-Aviv, Israel (institutional review board (IRB) no. 0066-20-MHS). The IRB includes an exemption from informed consent.
Reporting Summary. Further information on research design is available in the Nature Research Reporting Summary linked to this article.

\section{Data availability}

To protect patient privacy, underlying electronic health records may be accessed via a remote server pending a material transfer agreement. All other data are present in the paper.

\section{Acknowledgements}

This work was supported by the Israel Science Foundation (grant no. 3633/19 to

R. Kishony and G.C.) as part of the KillCorona-Curbing Coronavirus Research Program.

\section{Author contributions}

Study design: M.L.-T., I.Y., T.P., S.G., G.C. and R. Kishony. Data collection: M.L.-T., I.Y., L.S., E.H., R. Katz, T.W., V.N. and Z.G. Data analysis: M.L.-T., I.Y. and R. Kishony. Data interpretation: M.L.-T., I.Y., J.K., L.S., T.W., V.N., A.B.-T., T.P., S.G., G.C. and R. Kishony. Writing: M.L.-T., I.Y. and R. Kishony, with comments from all authors.

\section{Competing interests}

The authors declare no competing interests.

\section{Additional information}

Extended data are available for this paper at https://doi.org/10.1038/ s41591-021-01316-7.

Supplementary information The online version contains supplementary material available at https://doi.org/10.1038/s41591-021-01316-7.

Correspondence and requests for materials should be addressed to I.Y. or R.K.

Peer review information Nature Medicine thanks the anonymous reviewers for their contribution to the peer review of this work. Alison Farrell was the primary editor on this article and managed its editorial process and peer review in collaboration with the rest of the editorial team.

Reprints and permissions information is available at www.nature.com/reprints. 


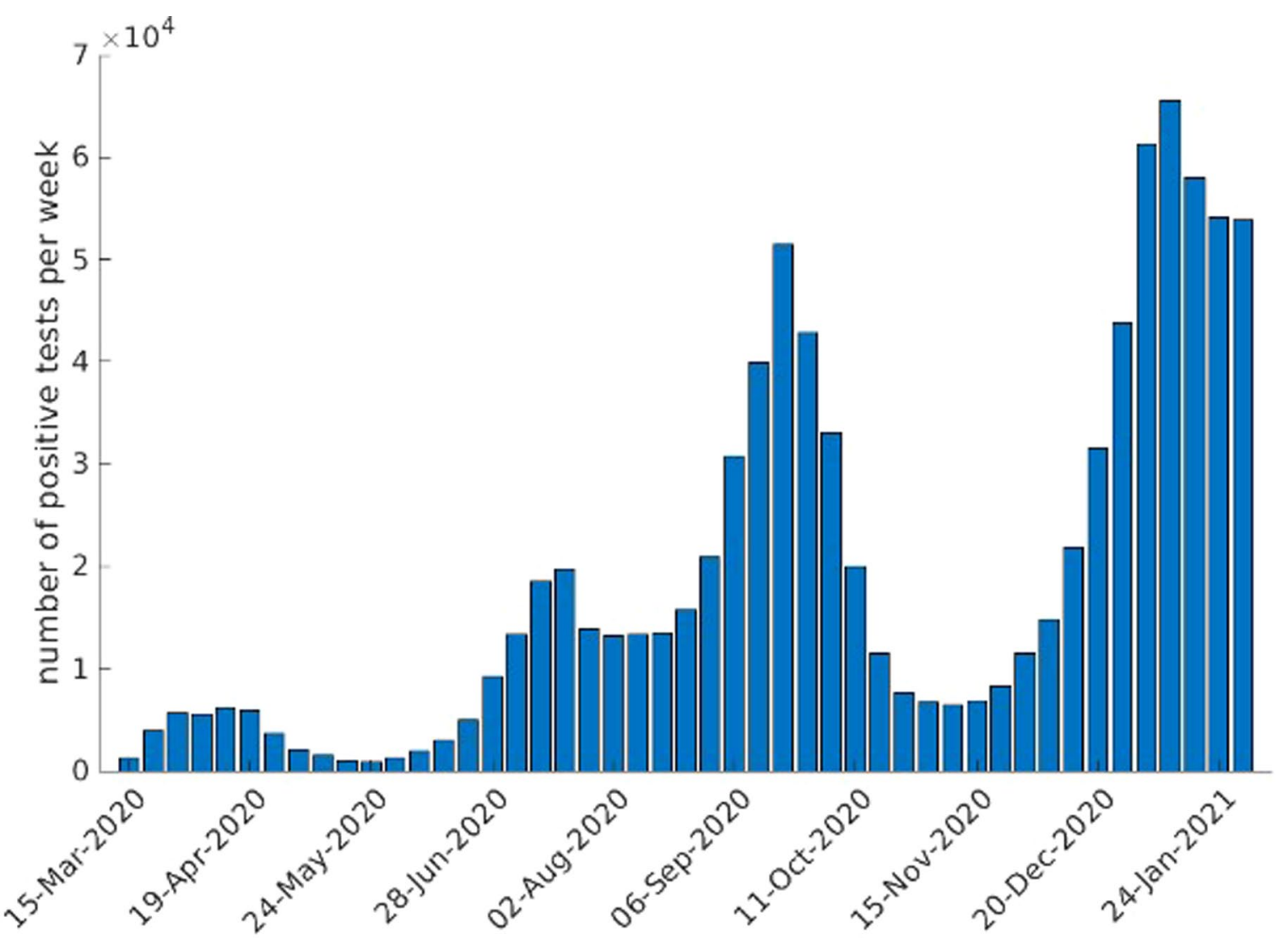

Extended Data Fig. 1 | Weekly positive COVID-19 tests in Israel. Total number of positive COVID-19 tests per week starting on March 15 th 2020 and updated till February 11th 2021. Data retrieved from Israel Ministry of Health website. 
a

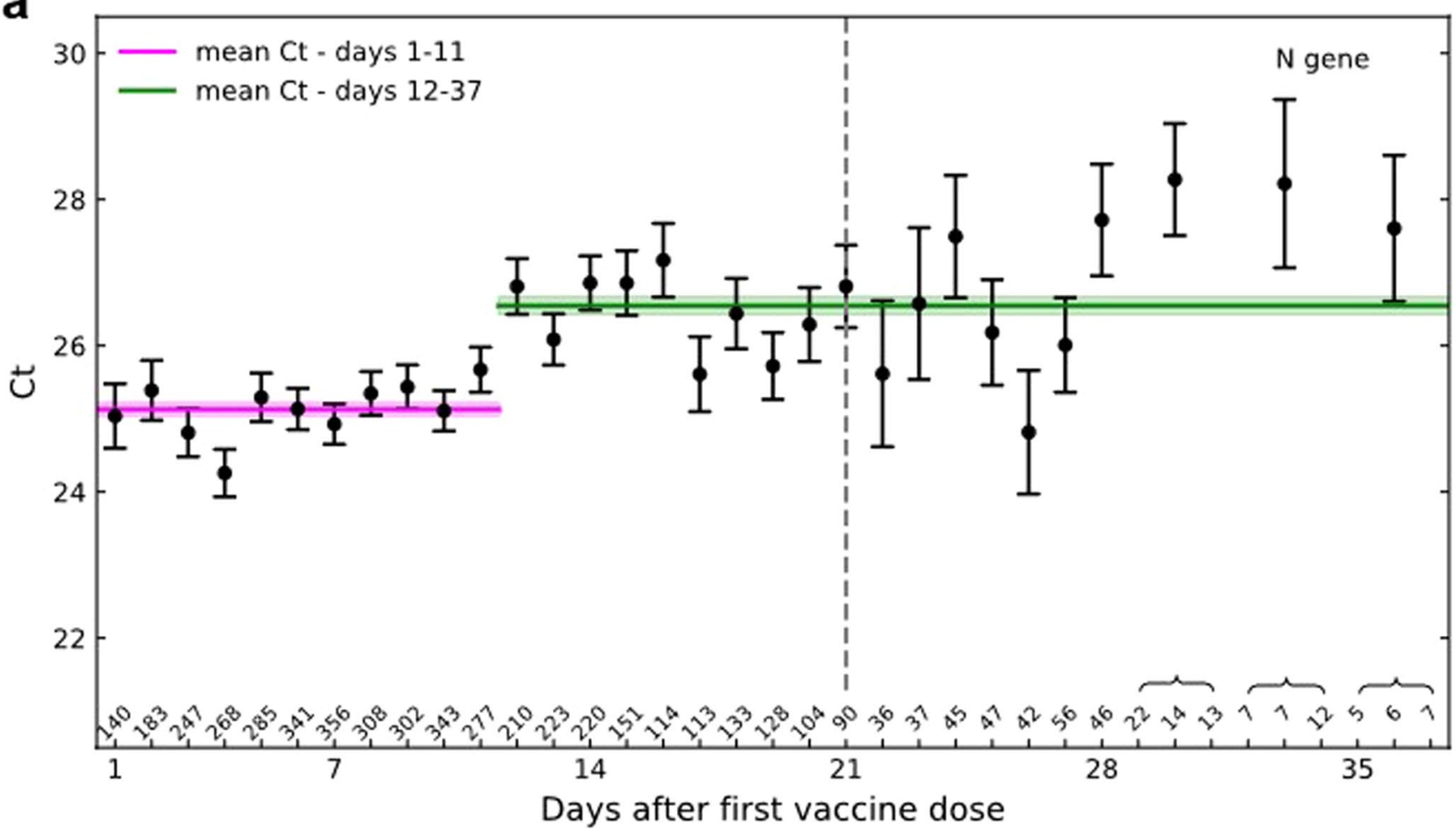

b

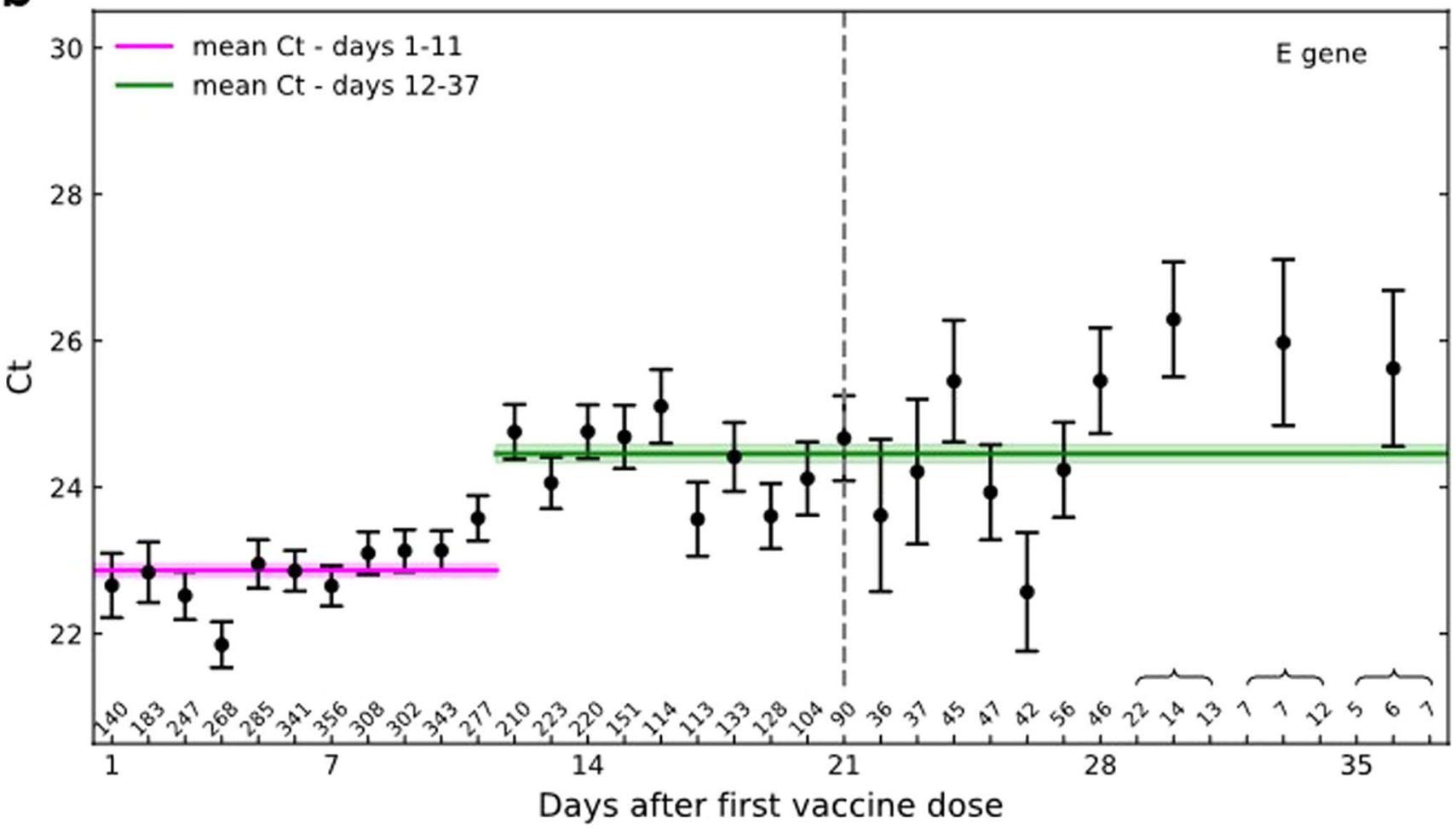

Extended Data Fig. 2 | Decreased SARS-CoV-2 viral load after 12 days post-vaccination. Mean Ct values of the $\mathrm{N}$ and E genes for positive tests following vaccination are plotted by the day the sample was taken $(n=4,938)$. Error bars indicate the standard error of the mean. For days $29-37$ each dot represents the mean of 3 consecutive days. a, N gene. b, E gene. For RdRp gene see Fig. 1. 

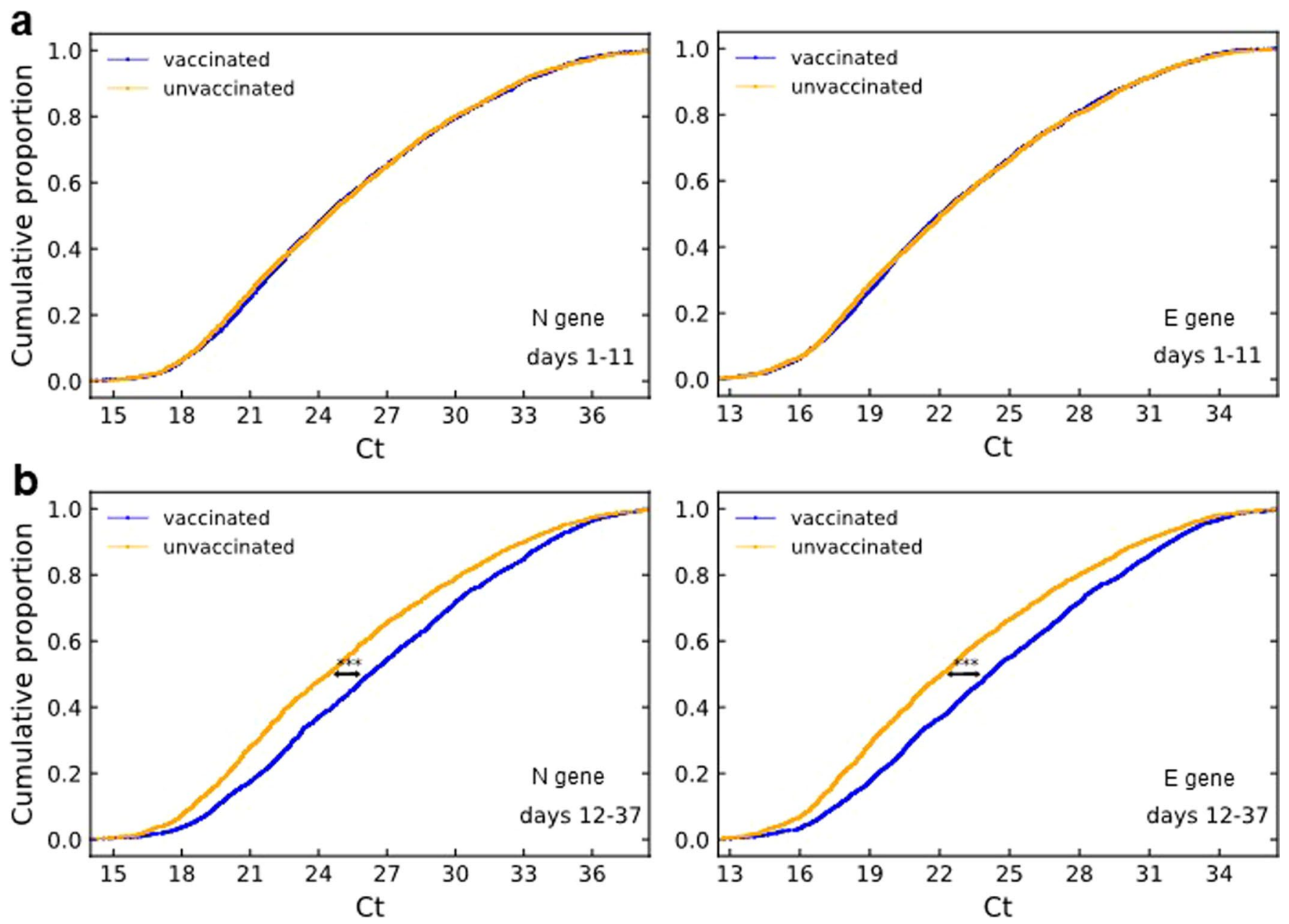

Extended Data Fig. 3 | Comparison of SARS-CoV-2 viral loads among patients vaccinated prior to positive sample and unvaccinated patients. The distribution of $\mathrm{Ct}$ values of viral genes as determined for positive samples taken either 1-11 days post-vaccination ( $n=3,050$, blue, top panels) or 12-37 days post vaccination ( $n=1,888$, blue, bottom panels) with their respective demographically-matched control groups (orange, ${ }^{\star \star \star}-P_{\text {-value }}<10^{-10}$, two-sided Mann-Whitney U test). a, 1-11 days post-vaccination. b, 12-37 days post-vaccination. For RdRp gene see Fig. 2a,b. 

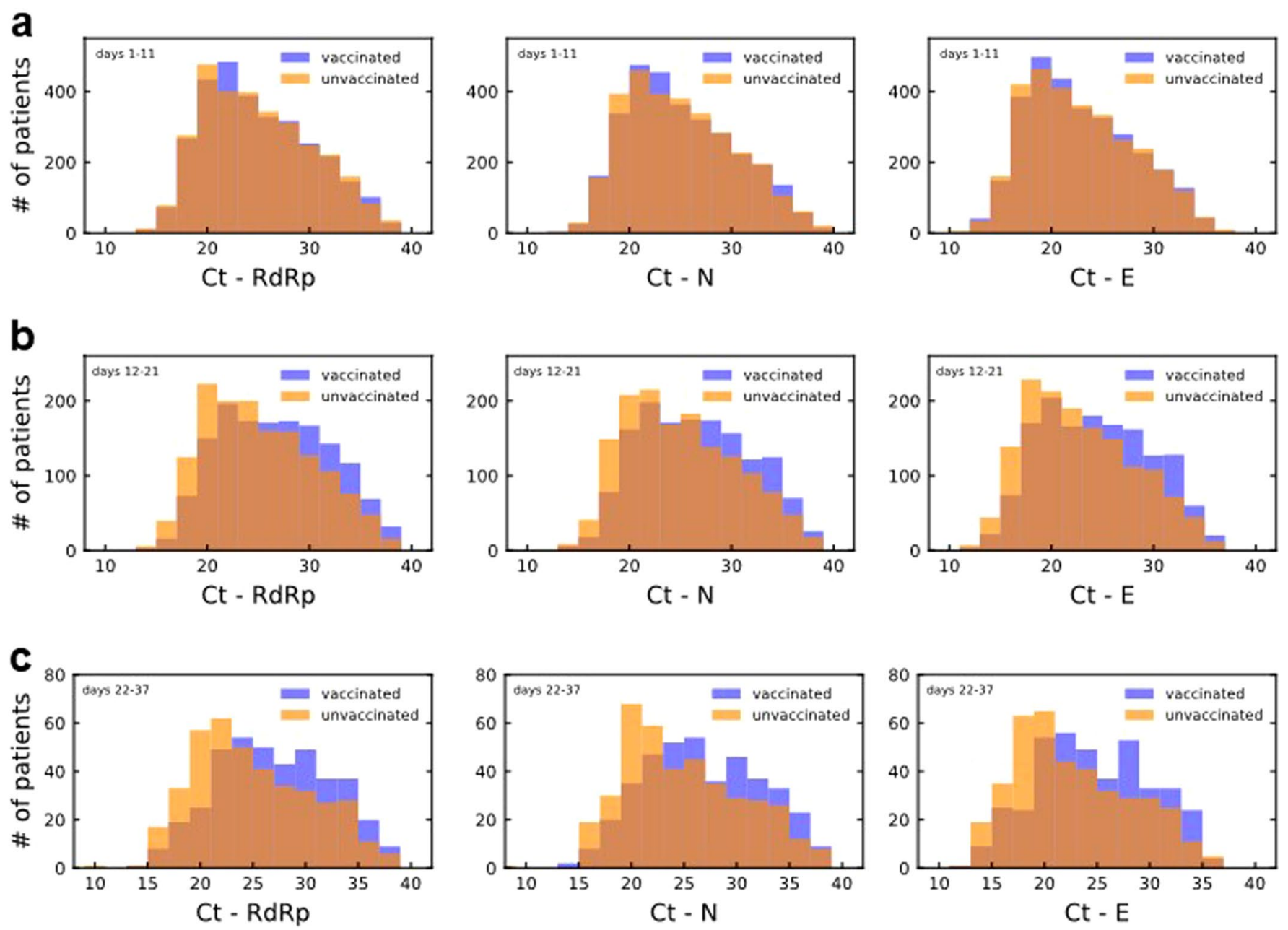

Extended Data Fig. 4 | SARS-CoV-2 viral load distribution for vaccinated and unvaccinated patients. The distribution of $\mathrm{Ct}$ values of viral genes as determined for positive samples taken a, 1-11 days post-vaccination with standard deviation of $5.27 \pm 0.07,5.27 \pm 0.07$ and $5.19 \pm 0.07(n=3,050), \mathbf{b}, 12-21$ days post-vaccination with standard deviation of $5.39 \pm 0.10,5.39 \pm 0.10$ and $5.33 \pm 0.10(n=1,486)$ and $\mathbf{c}, 22-37$ days post-vaccination with standard deviation of $5.38 \pm 0.19,5.47 \pm 0.19$ and $5.37 \pm 0.19(n=402)$ for RdRp, $N$ and E genes respectively. 
a

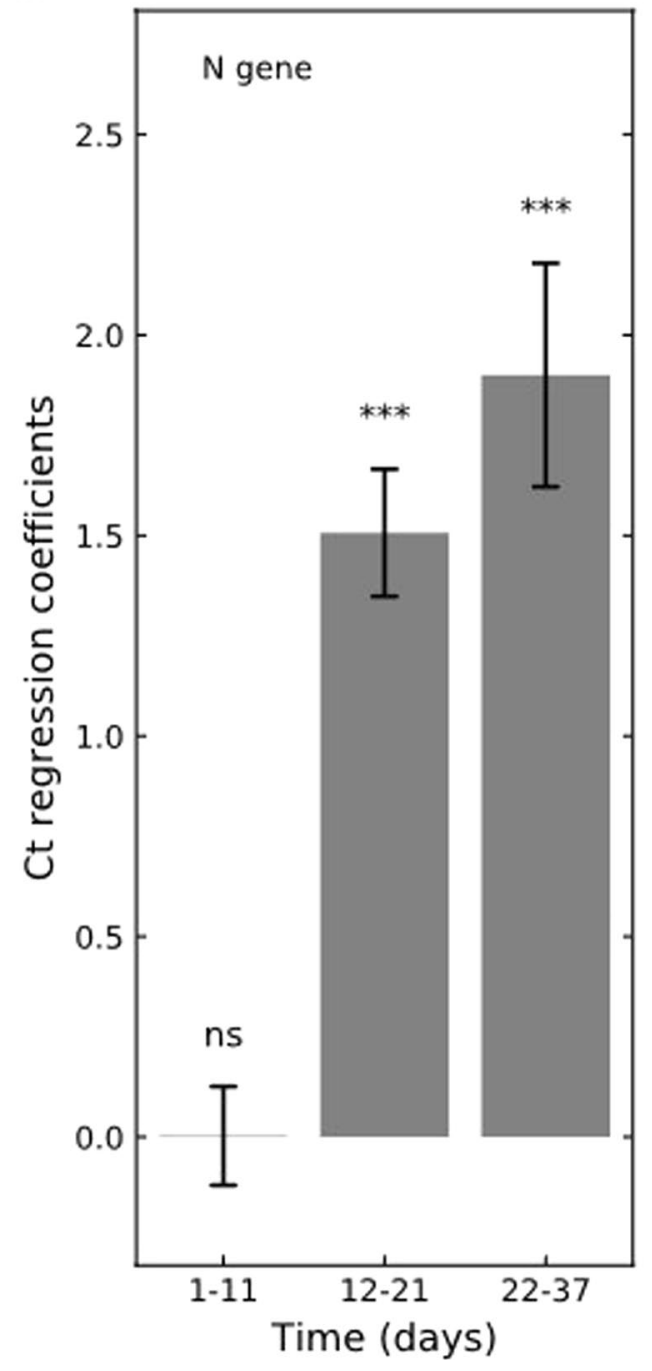

b

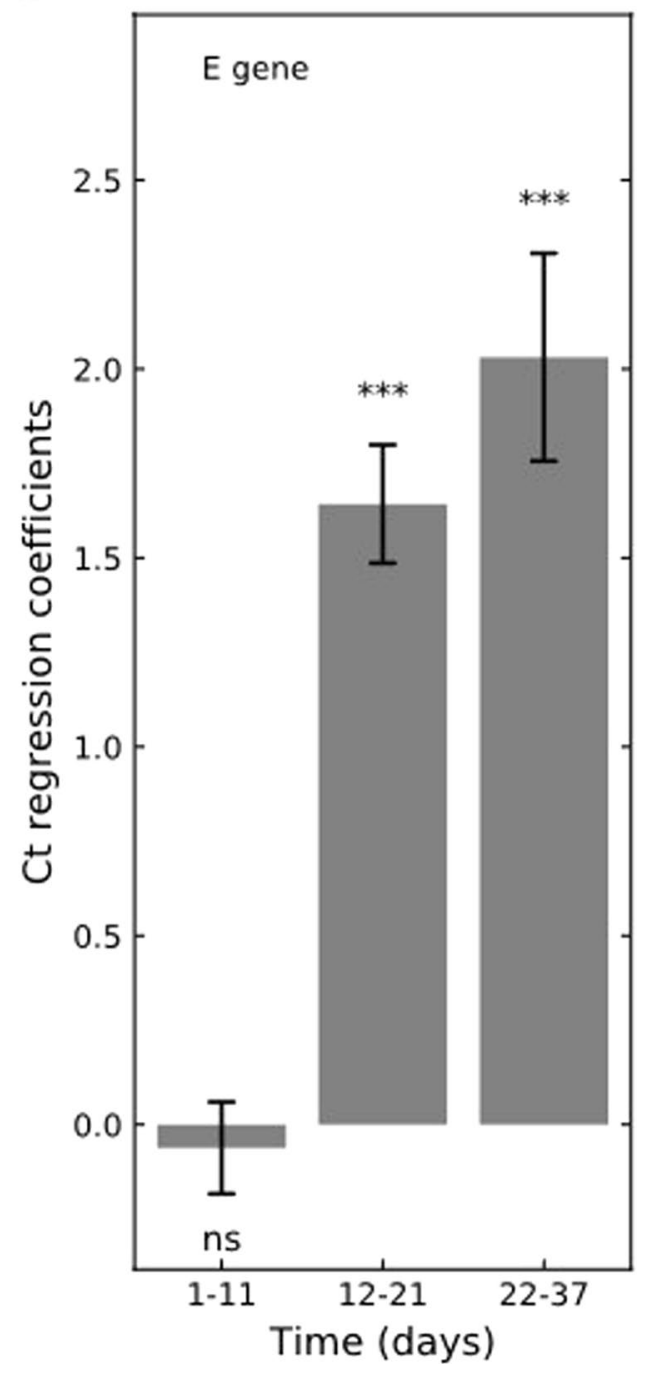

Extended Data Fig. $\mathbf{5}$ | Viral load is negatively associated with vaccination starting $\mathbf{1 2}$ days post-vaccination. The coefficient for the association of $\mathrm{Ct}$ of viral genes with time of vaccination in comparison to unvaccinated patients as identified in a single multivariable linear regression analysis accounting for age and sex (Methods, $n=9,876$ ). Error bars represent one standard deviation. ${ }^{\star}$ - P-value $<0.05$, ${ }^{\star \star}$ - P-value $<0.01$, ${ }^{\star \star \star}-\mathrm{P}$-value $<0.001$. $\mathbf{a}, \mathrm{N}$ gene, $\mathbf{b}, \mathrm{E}$ gene. For RdRp gene see Fig. 2c. 
a

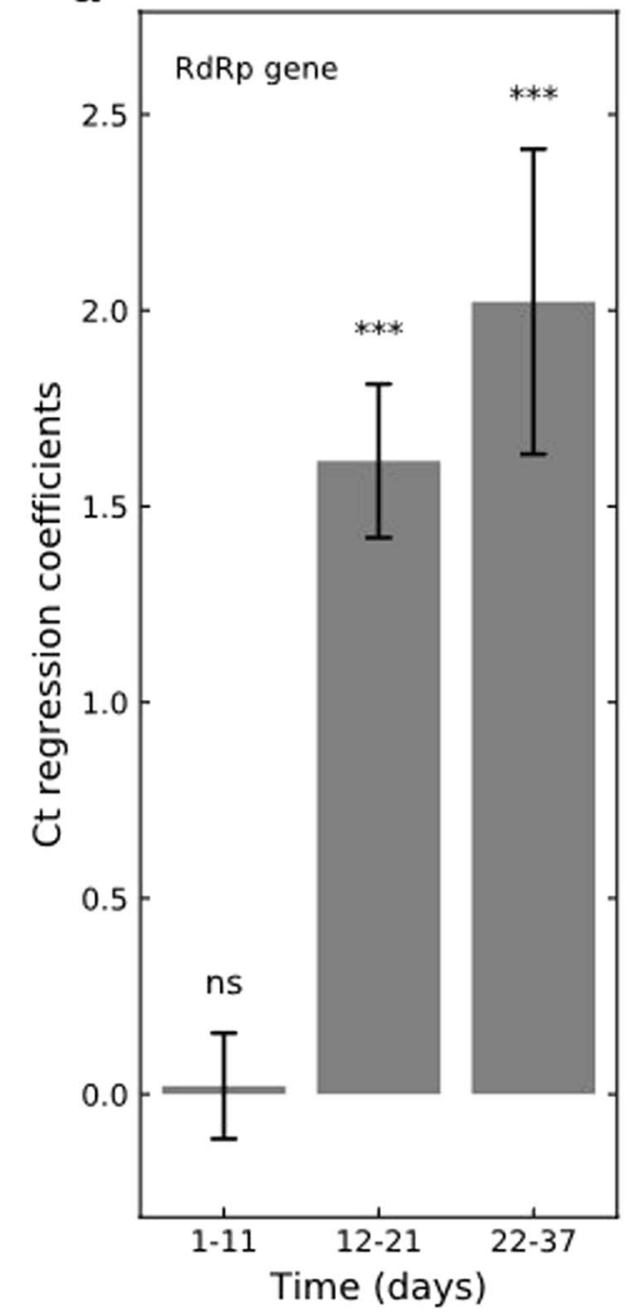

b

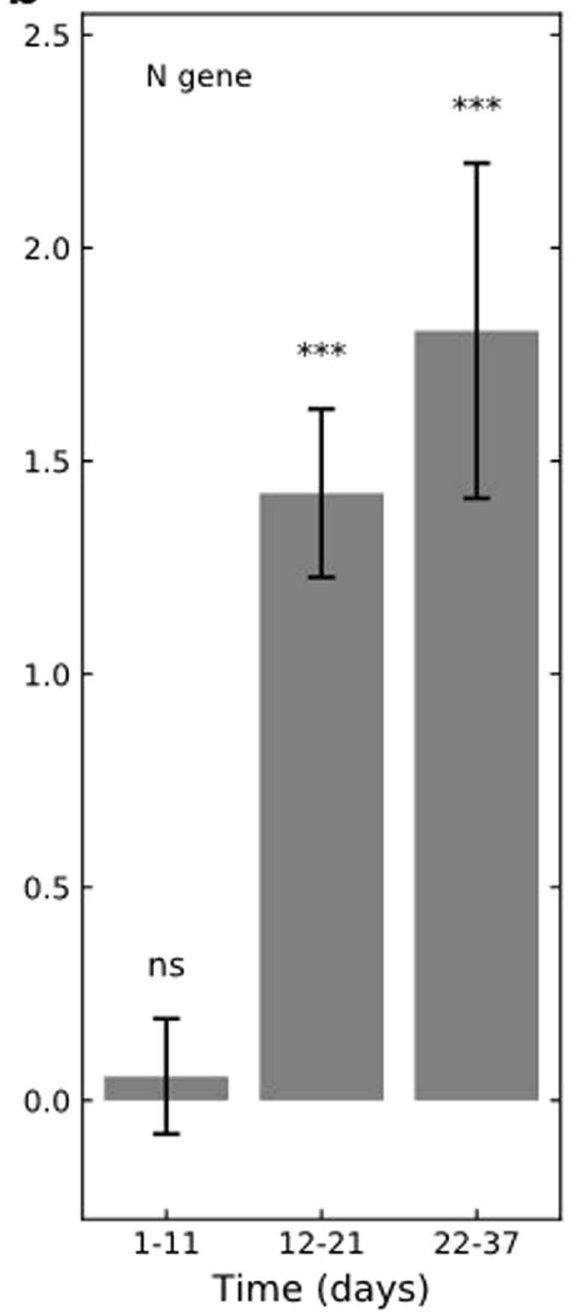

C

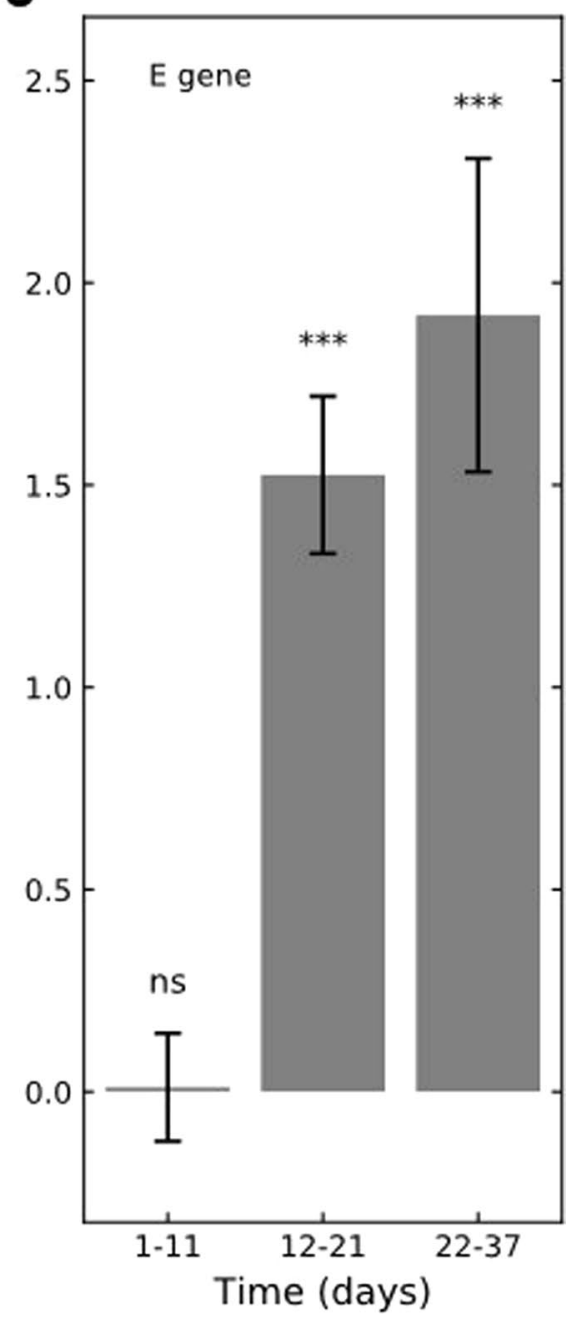

Extended Data Fig. 6 | Viral load is associated with vaccination also when applying an independent model for each time bin. The coefficient for the association of $\mathrm{Ct}$ of viral genes with time of vaccination in comparison to unvaccinated patients as identified in multivariable linear regression analyses accounting for age and sex for each time bin independently (Methods, $n=9,876$ ). Error bars represent one standard deviation. ${ }^{\star}-\mathrm{P}$-value $<0.05,{ }^{\star \star}-$ P-value $<0.01,{ }^{\star \star \star}$ - P-value $<0.001$. a, RdRp, b, N gene, $\mathbf{c}$, E gene. 
a

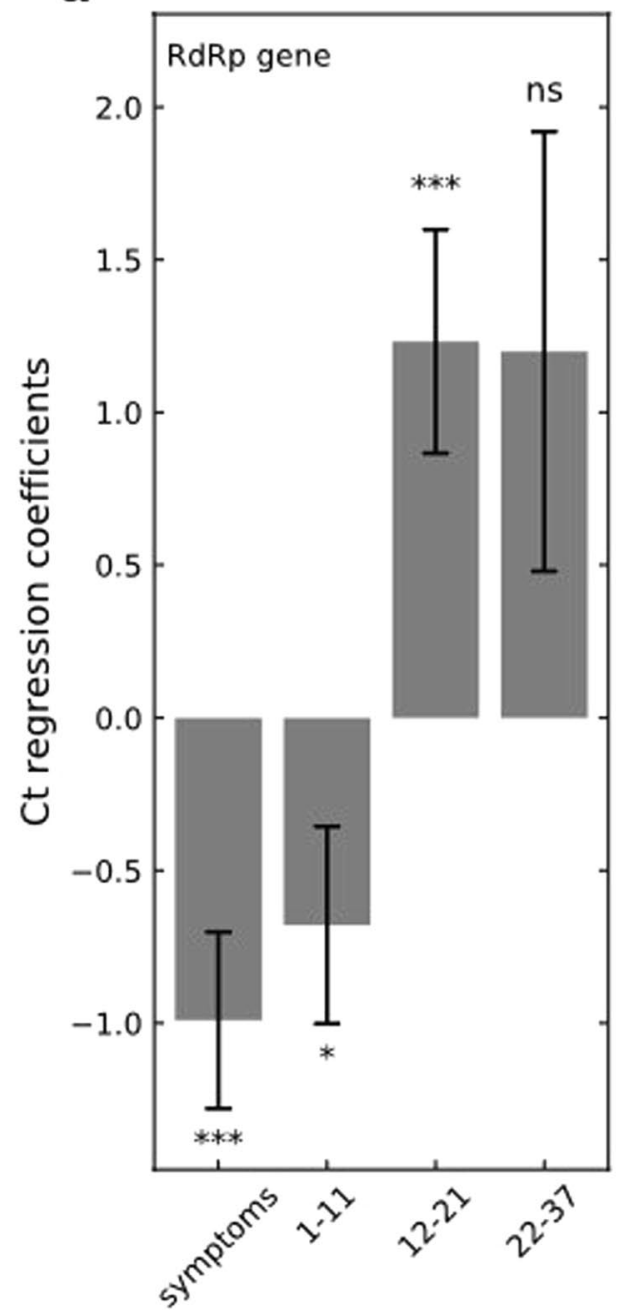

b

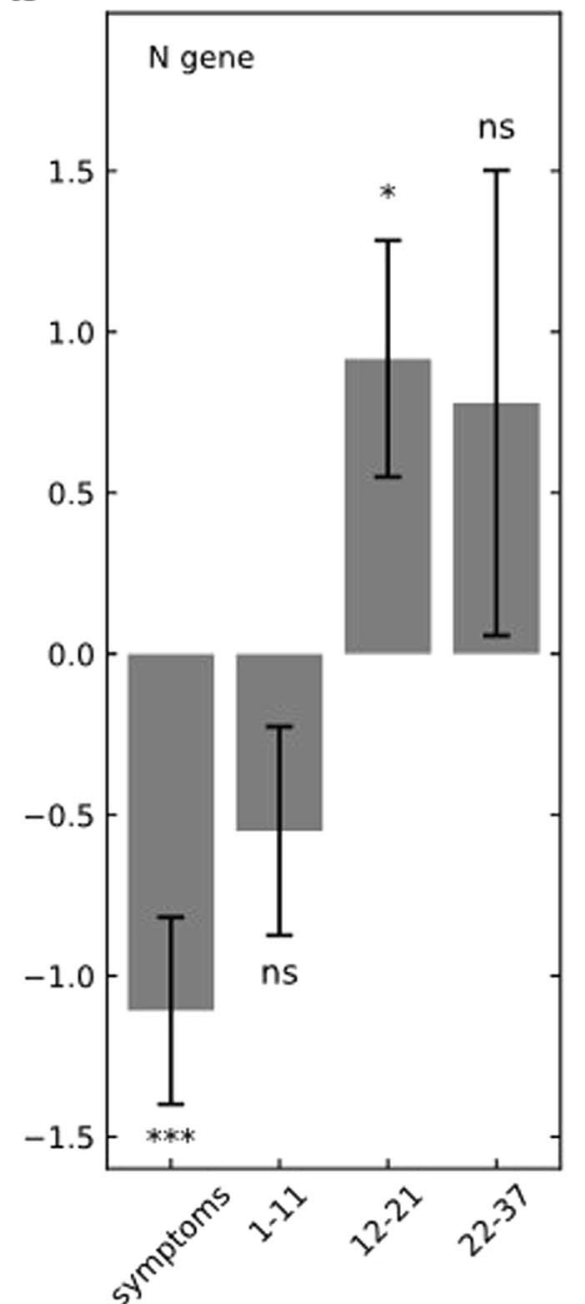

C

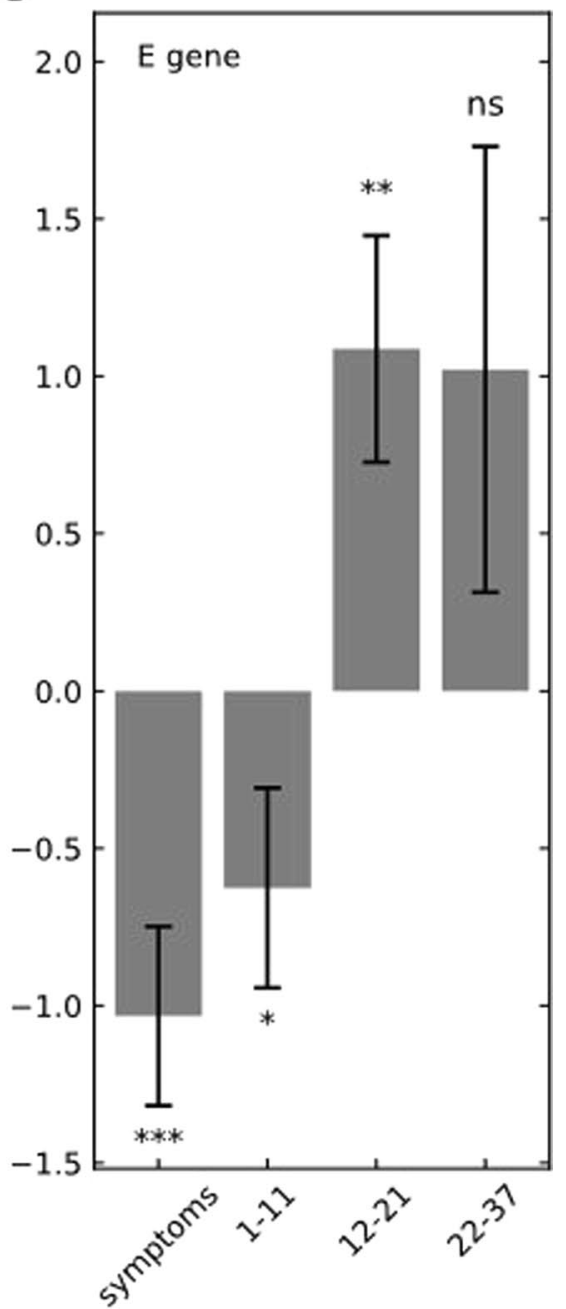

Extended Data Fig. 7 | Viral load is associated with vaccination when also accounting for symptomatic infections. The coefficient for the association of $\mathrm{Ct}$ of viral genes with time of vaccination in comparison to unvaccinated patients as identified in a single multivariable linear regression analysis accounting for age, sex and referral by symptoms for both vaccinated (symptomatic, $n=523$; asymptomatic, $n=260$ ) and unvaccinated (symptomatic, $n=534$; asymptomatic, $n=249$ ) patients (Methods). Error bars represent one standard deviation. ${ }^{\star}-P$-value $<0.05,{ }^{\star \star}-P$-value $<0.01,{ }^{\star \star \star}-P$-value $<$ 0.001. a, RdRp, b, N gene, $\mathbf{c}$, E gene. 


\section{Reporting Summary}

Nature Research wishes to improve the reproducibility of the work that we publish. This form provides structure for consistency and transparency in reporting. For further information on Nature Research policies, see our Editorial Policies and the Editorial Policy Checklist.

\section{Statistics}

For all statistical analyses, confirm that the following items are present in the figure legend, table legend, main text, or Methods section.

$\mathrm{n} / \mathrm{a}$ Confirmed

$\bigotimes$ The exact sample size $(n)$ for each experimental group/condition, given as a discrete number and unit of measurement

Х $\square$ A statement on whether measurements were taken from distinct samples or whether the same sample was measured repeatedly

The statistical test(s) used AND whether they are one- or two-sided

Only common tests should be described solely by name; describe more complex techniques in the Methods section.

$\bigotimes$ A description of all covariates tested

\ A description of any assumptions or corrections, such as tests of normality and adjustment for multiple comparisons

A full description of the statistical parameters including central tendency (e.g. means) or other basic estimates (e.g. regression coefficient) AND variation (e.g. standard deviation) or associated estimates of uncertainty (e.g. confidence intervals)

For null hypothesis testing, the test statistic (e.g. $F, t, r$ ) with confidence intervals, effect sizes, degrees of freedom and $P$ value noted Give $P$ values as exact values whenever suitable.

Х $\square$ For Bayesian analysis, information on the choice of priors and Markov chain Monte Carlo settings

Х $\square$ For hierarchical and complex designs, identification of the appropriate level for tests and full reporting of outcomes

$\triangle \square$ Estimates of effect sizes (e.g. Cohen's $d$, Pearson's $r$ ), indicating how they were calculated

Our web collection on statistics for biologists contains articles on many of the points above.

\section{Software and code}

Policy information about availability of computer code

Data collection SQL query.

Data analysis Python. Including: scipy (v1.1.0) and statsmodels (v0.9.0).

For manuscripts utilizing custom algorithms or software that are central to the research but not yet described in published literature, software must be made available to editors and

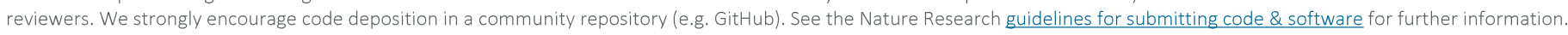

\section{Data}

Policy information about availability of data

All manuscripts must include a data availability statement. This statement should provide the following information, where applicable:

- Accession codes, unique identifiers, or web links for publicly available datasets

- A list of figures that have associated raw data

- A description of any restrictions on data availability

A list of figures with associated raw data: Figure 1, Figure 2, Extended data figures 1, 2 and 3.

To protect patient privacy, underlying electronic health records may be accessed via a remote server pending MTA. 
Please select the one below that is the best fit for your research. If you are not sure, read the appropriate sections before making your selection.

\ Life sciences

Behavioural \& social sciences

Ecological, evolutionary \& environmental sciences

For a reference copy of the document with all sections, see nature.com/documents/nr-reporting-summary-flat.pdf

\section{Life sciences study design}

All studies must disclose on these points even when the disclosure is negative.

Sample size Sample size was not restricted. It was only limited by the number of patients with available positive post-vaccination RT-qPCR test data.

Data exclusions We have excluded patients above 90 years old of age. Since our analysis required matching unvaccinated patients for each vaccinated one with several conditions, we could not find matched patients for this small age group.

Replication We report the results of an observational study.

Randomization As a control group, the same number of patients as the original sample were chosen randomly while maintaining demographic characteristics, namely age and sex.

Blinding We report the results of an observational study.

\section{Reporting for specific materials, systems and methods}

We require information from authors about some types of materials, experimental systems and methods used in many studies. Here, indicate whether each material, system or method listed is relevant to your study. If you are not sure if a list item applies to your research, read the appropriate section before selecting a response.

Materials \& experimental systems

$\mathrm{n} / \mathrm{a}$ Involved in the study

Х $\square$ Antibodies

Methods

\ $\square$ Eukaryotic cell lines

\ $\square$ Palaeontology and archaeology

$\mathrm{n} / \mathrm{a}$ Involved in the study

X $\square$ Chip-seq

X $\square$ Flow cytometry

\ $\square$ Animals and other organisms

Х $\square$ MRI-based neuroimaging

$\square \bigotimes$ Human research participants

\ $\square$ Clinical data

\ $\square$ Dual use research of concern

\section{Human research participants}

Policy information about studies involving human research participants

Population characteristics

Recruitment

Ethics oversight

\section{Population is described in Extended Data Table 1.}

This is an observational study involving electronic health records analysis.

Ethics committee of Maccabi Healthcare Services. IRB: 0066-20-MHS.

Note that full information on the approval of the study protocol must also be provided in the manuscript. 